\title{
Antidiabetic effect of long-term supplementation with Siraitia grosvenori on the spontaneously diabetic Goto-Kakizaki rat
}

\author{
Yasushi A. Suzuki ${ }^{1 *}$, Mayuko Tomoda ${ }^{1}$, Yuji Murata ${ }^{1,2}$, Hiroshi Inui ${ }^{2,3}$, Masaki Sugiura ${ }^{1,2}$ \\ and Yoshihisa Nakano,3 \\ ${ }^{1}$ Biochemical Laboratory, Saraya Company Ltd, Kashiwara, Osaka 582-0028, Japan \\ ${ }^{2}$ Department of Applied Biological Chemistry, Osaka Prefecture University, Sakai, Osaka 599-8531, Japan \\ ${ }^{3}$ Center for Research and Development of Bioresources, Osaka Prefecture University, Sakai, Osaka 599-8570, Japan
}

(Received 12 April 2006 - Revised 2 October 2006 - Accepted 10 October 2006)

\begin{abstract}
Siraitia grosvenori Swingle (SG) is a traditional Chinese fruit used as a folk medicine. Its extract (SG-ex) contains potent sweet elements with a sweetness several hundred times higher than table sugar. We investigated the antidiabetic effect of SG-ex in the type 2 diabetic Goto-Kakizaki (GK) rat. Diabetic 7-week-old GK rats were fed a diet supplemented with $0.4 \%$ of the SG-ex for 13 weeks, and its antidiabetic effects were evaluated. SG-ex had no effect on food intake or body weight. In oral glucose tolerance tests (OGTT), SG-ex supplementation improved the insulin response at $15 \mathrm{~min}$ (control, 63 (SEM 6) pM; SG-ex, 107 (SEM 20) pM; $P<0.05)$ and reduced the plasma glucose level at 120 min after the glucose administration (control, 18.5 (SEM 0.8) mM; SG-ex, 14.8 (SEM 0.7) mM; $P<0.05$ ). The total amount of insulin in whole pancreas taken from fasting rats was higher in the SG-ex-supplemented group, which may explain the greater capacity to secrete insulin during the OGTT. Thiobarbituric acidreactive substances in both the liver and the plasma were lower in the SG-ex-supplemented group, suggesting that an absorbable component in SGex has an antioxidative effect on lipid peroxidation, thereby counteracting the oxidative stress caused by a diabetic state. Excreted urine volume and urinary albumin level for $24 \mathrm{~h}$ were both reduced in the SG-ex-supplemented group, suggesting the attenuation of kidney damage that is caused by diabetes. These data indicate that SG-ex supplementation may prevent complications and attenuate pathological conditions for type 2 diabetes, along with its sweet characteristics.
\end{abstract}

Siraitia grosvenori Swingle: Antidiabetic effects: Diabetes: Insulin response: Sugar substitutes

Siraitia grosvenori Swingle (SG) is a traditional Chinese fruit, and belongs to Cucurbitaceous species. It has been used as a folk medicine for sore throats, coughs, and minor stomach and intestinal troubles. The extract from this fruit (SG-ex) has been reported to have a sweetness 150 times as strong as sucrose in spite of having minimal energy content (Lee, 1975). Its dry fruit has been found to contain several triterpene glycosides, namely mogroside IV, mogroside V and mogroside VI (Takemoto et al. 1983). Two additional triterpene glycosides in SG-ex, siamenoside I and 11-oxo-mogroside V (11OM-V), were also found to have a strong sweetness (Kasai et al. 1989). The relative sweetness of mogroside IV, mogroside V, siamenoside I and 110M-V were 392, 425, 563, and eighty-four times as potent as that of sucrose, respectively (Matsumoto et al. 1990). Because of this potent sweetness with a minimal energy content, SG-ex has been commercially utilised as a sweet component in sugar substitutes, especially for diabetes.

Recently, SG-ex or its constituents have been shown to have various physiological functions. For instance, 11OM-V has a strong inhibitory effect on LDL oxidation, and thus is likely to reduce the atherogenic potential of LDL (Takeo et al. 2002).
The inhibitory effects of SG-ex on the initiation and promotion of cancer have also been reported (Takasaki et al. 2003). In addition, SG-ex has been found to have anti-allergenic effects (Hossen et al. 2005). We also found that SG-ex reduced hyperglycaemia after a single oral administration of maltose in the rat (Suzuki et al. 2005). In vitro, SG-ex, as well as its constituents mogroside V, mogroside IV, mogroside III and siamenoside I, have been found to inhibit $\alpha$-glucosidase (Suzuki et al. 2005). The $\alpha$-glucosidase inhibitors are known to delay carbohydrate digestion in the small-intestinal tract and thereby to reduce rises of postprandial plasma glucose and of plasma insulin levels (Clissold \& Edwards, 1988; Toeller, 1994). Voglibose and acarbose are well-known $\alpha$-glucosidase inhibitors and in fact have been shown to be beneficial for treating type 2 diabetes as drugs (Saito et al. 1998; Rury et al. 1999). We thus hypothesised that long-term administration of SG-ex is beneficial for type 2 diabetes.

The Goto-Kakizaki (GK) rat is an animal model of spontaneous non-insulin-dependent diabetes mellitus (Goto et al. 1975). The diabetic state was generated by selective breeding repeated over many generations with glucose intolerance as a selection index, starting from a colony of non-diabetic Wistar

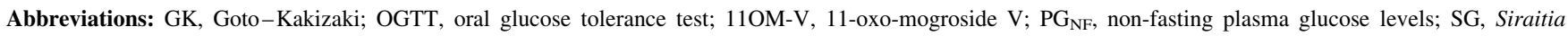
grosvenori Swingle; SG-ex, Siraitia grosvenori Swingle extract; TBARS, thiobarbituric acid-reactive substance.

* Corresponding author: Dr Yasushi A. Suzuki, fax +81 72977 2224; email suzuki-y@saraya.com
} 
rats (Goto \& Kakizaki, 1981). The pathogenesis of diabetes in the GK rat includes an impaired insulin secretion (Portha et al. 1991), insulin resistance (Bisbis et al. 1993) and abnormal glucose metabolism (Ostenson et al. 1993). In contrast to many other rodent models of type 2 diabetes (Janssen et al. 1999), GK rats do not become obese (O'Rourke et al. 1997) and do not develop hyperlipidaemia (Zhou et al. 1995). These characteristics are similar to the typical Asian-type diabetes, and thus we found it an appropriate model to study the effect of SG-ex on type 2 diabetes.

Accordingly, the present study was performed to examine the effect of SG-ex supplementation in the diet on attenuating the pathological status of type 2 diabetes.

\section{Materials and methods}

\section{Preparation of the extract from Siraitia grosvenori}

S. grosvenori (SG-ex) was prepared in Guilin S\&T New Tech Company (Guilin, China) as described previously (Suzuki et al. 2005). Briefly, fresh fruits of $S$. grosvenori were crushed and boiled in water and the water-soluble fraction was concentrated until soluble solids of a 64.0 Brix paste, measured by refractometry at $20^{\circ} \mathrm{C}$. Mogroside $\mathrm{V}, 11 \mathrm{OM}-\mathrm{V}$, mogroside IV, mogroside III and siamenoside I contents in SG-ex determined by HPLC method (Suzuki et al. 2005) were approximately $2 \cdot 1,0.2,0 \cdot 8,0.7$ and $0.3 \%$, respectively.

\section{Study design}

Male GK rats (aged 5 weeks) were obtained from Clea Japan (Osaka, Japan). The animals were kept on a standard pellet diet (CE-2; Clea Japan, Osaka, Japan) and water ad libitum to acclimatise to their environment for 1 week. The rats were fed the control artificial diet (Table 1) for another week, then randomly allocated into two groups; control and SG-ex groups. They were housed individually at controlled temperature $\left(23 \pm 2^{\circ} \mathrm{C}\right)$, humidity $(60 \pm 10 \%)$ and lighting (09.00 to 21.00 hours), and allowed free access to the designated artificial diet and water for the subsequent 13 weeks. The content of the designated artificial diet is shown in Table 1. Food intake and body weight were measured every other day. Average food intake was about $15 \mathrm{~g} / \mathrm{d}$ in both groups. Excess amounts of diet $(25 \mathrm{~g} / \mathrm{d})$ were given at 09.00 hours every day. Blood was collected in non-fasting conditions at 10.00 hours every other

Table 1. Ingredients of the experimental diets

\begin{tabular}{lc}
\hline Ingredient & Content $(\mathrm{g} / \mathrm{kg})$ \\
\hline Maize starch & 530 \\
Milk casein & 200 \\
Sucrose & 100 \\
Soyabean oil & 70 \\
AIN-93VX mineral mix & 35 \\
AIN-93G vitamin mix & 10 \\
Methionine & 3 \\
Choline chloride & 2 \\
Cellulose & 46 \\
Siraitia grosvenori Swingle extract & 4 \\
Total & 1000 \\
\hline
\end{tabular}

${ }^{*}$ For the control, Siraitia grosvenori Swingle extract was replaced by cellulose. week from the tail vein into heparinised tubes, and centrifuged at $3000 \mathrm{~g}$ for $10 \mathrm{~min}$ at $4^{\circ} \mathrm{C}$. The supernatant fraction (plasma) was collected and stored at $-20^{\circ} \mathrm{C}$ until analysed. Plasma glucose levels were measured by a glucose oxidase method using a commercial kit (Glucose B-test Wako; Wako Pure Chemical Industries, Osaka, Japan). At week 13 of treatment (age 20 weeks) after $16 \mathrm{~h}$ starvation, rats were anaesthetised with diethyl ether and killed. Blood was collected from the vena cava, and the heart, liver, kidney, spleen, pancreas and small intestine tissues were collected, weighed, frozen in liquid $\mathrm{N}_{2}$ and stored at $-80^{\circ} \mathrm{C}$ until analysed. The animals used were maintained in accordance with the guidelines of the National Research Council (1985).

\section{Oral glucose tolerance test}

An oral glucose tolerance test (OGTT) was performed at week 7 of treatment (age 14 weeks). The tail vein blood was collected in heparinised microtubes after $16 \mathrm{~h}$ starvation as a control blood sample at $0 \mathrm{~min}$. Glucose $(1 \mathrm{~g} / \mathrm{kg}$ body weight) was intubated orally, and the tail vein blood was collected in a heparinised microtube at 30, 60, 90 and 120 min after the intubation. Plasma glucose levels and plasma insulin levels (ELISA kit, rebis insulin-rat U type; Shibayagi, Gunma, Japan) were determined for each time point.

\section{Urine analysis}

During weeks 11 and 12 of supplementation (age 18-19 weeks), urine was collected. A rat was individually placed in a metabolism cage for $3 \mathrm{~d}$ before the urine collection in order to acclimatise the rat to the environment. The rat could freely access the water and food. The urine was collected at $24 \mathrm{~h}$ intervals for $2 \mathrm{~d}$. Urinary albumin levels were determined by a sandwich ELISA kit (Shibayagi, Gunma, Japan).

\section{Pancreatic insulin level}

Fasting pancreatic insulin levels were determined by a sandwich ELISA kit (Shibayagi, Gunma, Japan). Briefly, the pancreas $(150 \mathrm{mg})$ was homogenised in $600 \mu \mathrm{l}$ PBS containing $0.05 \%$ Triton X-100 by a polytron homogeniser. The homogenate was serially diluted with the buffer provided in the ELISA kit, and the insulin level was measured according to the instructions.

\section{Thiobarbituric acid-reactive substance level}

Thiobarbituric acid-reactive substance (TBARS) levels of the liver, kidney, pancreas and plasma were determined (Ohkawa et al. 1979). Briefly, each tissue (liver, kidney, pancreas; $150 \mathrm{mg}$ ) was homogenised in $600 \mu \mathrm{KCl}(1.15 \%)$ solution by a polytron homogeniser. SDS $(40 \mu \mathrm{l} ; 8.1 \%), 300 \mu \mathrm{l}$ sodium acetate buffer (20\%; pH 3.5) and $300 \mu$ l thiobarbituric acid $(0.8 \%)$ were added to $80 \mu \mathrm{l}$ of the homogenate or $80 \mu \mathrm{l}$ of the plasma, and incubated at $95^{\circ} \mathrm{C}$ for $20 \mathrm{~min}$. After cooling down the reaction mixture to room temperature, $200 \mu \mathrm{l}$ of the deionised water and $1 \mathrm{ml}$ of the $\mathrm{n}$-butanol-pyridine mixture $(15: 1 ; \mathrm{v} / \mathrm{v})$ were added, mixed and centrifuged at $4000 \mathrm{rpm}$ for $10 \mathrm{~min}$. The absorbance of the supernatant fraction 
was measured at $535 \mathrm{~nm}$ in a spectrophotometer. 1,1,3,3-Tetraethoxypropane $(0.5,1.25,2.5 \mathrm{nmol} / 80 \mu \mathrm{l})$ was used as a standard. Protein concentrations for these tissues were determined by a BCA assay (Pierce, Rockford, IL, USA).

\section{Plasma analysis}

Plasma TAG (triglyceride E-test), plasma cholesterol (cholesterol E-test), transaminase (transaminase C II-test), $\gamma$-glutamyl transpeptidase ( $\gamma$-glutamyl transpeptidase $\mathrm{C}$-test), lactase dehydrogenase (lactate dehydrogenase C II-test) and alkaline phosphatase (alkaline phospha $\mathrm{K}$ kit) were determined according to the manufacturer's instructions (Wako Pure Chemical Industries, Osaka, Japan).

\section{Statistical analysis}

Data on OGTT were analysed by two-way ANOVA for repeated measures, and post hoc analyses were done by Fisher's least significant difference test. These statistical analyses were performed with GB-Stat 5.4 (Dynamic Microsystems, Silver Spring, MD, USA). Student's $t$ test was performed for urine, pancreatic insulin, TBARS and plasma analysis. Results are expressed as mean values with their standard errors. Statistical significance was defined as $P<0 \cdot 05$.

\section{Results}

Food intake, body weight, tissue weight and non-fasting plasma glucose

There were no significant differences between the control and the SG-ex-supplemented rats for both food intake and body weight (data not shown), suggesting that SG-ex at this dose for a 13-week supplementation period does not have any adverse effects on feeding performance. Average food intake was about $15 \mathrm{~g} / \mathrm{d}$ for both groups. The non-fasting plasma glucose levels $\left(\mathrm{PG}_{\mathrm{NF}}\right)$ were measured every other week (Fig. 1). The $\mathrm{PG}_{\mathrm{NF}}$ were almost identical between the two groups at the beginning of the study until 4 weeks of treatment. However, at week 8 of treatment, the $\mathrm{PG}_{\mathrm{NF}}$ in the SG-ex group became lower than that in the control group, and this trend was maintained until the end of the study. Although the difference did not reach statistical significance $(P>0 \cdot 05)$, this result suggests

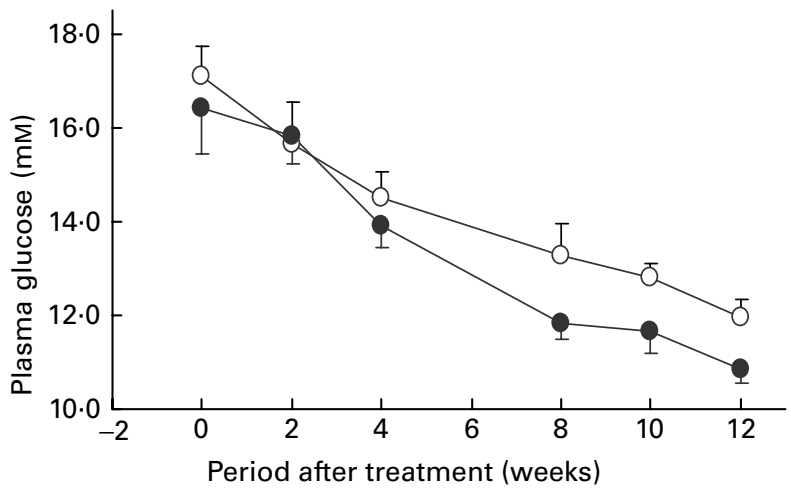

Fig. 1. Plasma glucose levels of Goto-Kakizaki rats in the non-fasting state. (๑), Siraitia grosvenori-extract-supplemented group; (○), control group. Values are means ( $n 10)$, with standard errors represented by vertical bars. that the longer supplementation with SG-ex may attenuate the elevated $\mathrm{PG}_{\mathrm{NF}}$ caused by diabetes.

\section{Oral glucose tolerance test}

OGTT were performed after 7 weeks of treatment. Fasting plasma glucose was identical between the control and the SG-ex-supplemented group (Fig. 2 (A)). The fluctuation patterns of plasma glucose levels after the glucose administration were similar between the two groups until $60 \mathrm{~min}$. The control group exhibited the typical pattern for diabetes: glucose administration caused a quick enhancement of plasma glucose and it stayed high once increased. It was, however, found to be significantly lower $(P<0 \cdot 05)$ at $120 \mathrm{~min}$ in the SG-ex group than in the control group, which suggests that orally supplemented SG-ex may improve the ability to control postprandial plasma glucose levels. The fasting plasma insulin level
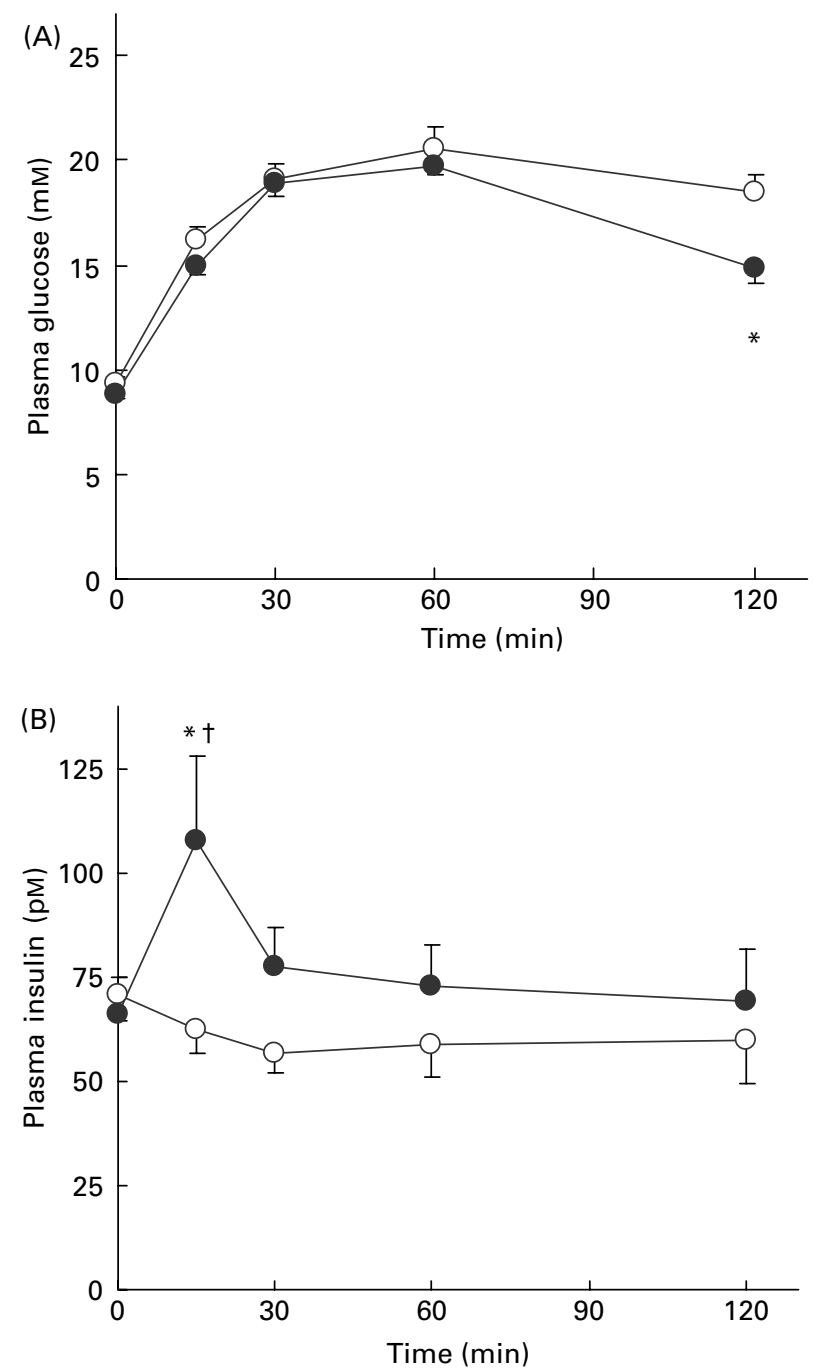

Fig. 2. Results of the oral glucose tolerance test (OGTT). Plasma glucose levels (A) and plasma insulin levels (B) at 0, 15, 30, 60 and 120 min after glucose administration in the OGTT. (๑), Siraitia grosvenori-extract-supplemented group; $(\bigcirc)$, control group. Values are means $(n 6)$, with standard errors represented by vertical bars. *Mean value was significantly different from that of the control group at the same time point $(P<0.05)$. †Mean value was significantly different from that at $0 \mathrm{~min}(P<0.05)$. 
was similar in both groups (Fig. 2 (B)). Strikingly, the SG-ex group showed a marked, short-lived increase at $15 \mathrm{~min}$ in the plasma insulin levels that returned to the control levels after $30 \mathrm{~min}$. This marked rise of plasma insulin could influence liver and peripheral tissues to facilitate glucose uptake and result in the decrease of plasma glucose levels at $120 \mathrm{~min}$ in the SG-ex group. Furthermore, these results also suggest that GK rats supplemented with SG-ex ameliorate the pancreatic insulin storage capacity during fasting conditions or the insulin-releasing capacity from the pancreas. We thus examined the fasting pancreatic insulin contents.

\section{Pancreatic insulin content}

The total amount of insulin in whole pancreas taken from fasting rats is shown in Fig. 3. As we had expected, pancreatic insulin contents were significantly higher in the SG-ex group than in the control group $(P=0 \cdot 013)$, suggesting that the pancreas is capable of storing more insulin in SG-ex-supplemented rats, which can then be released promptly responding to the rise in plasma glucose levels and properly regulate plasma glucose levels.

\section{Urine analysis}

The volume of urine and the urinary albumin levels are shown in Fig. 4. The excreted urine volume was significantly lower in the SG-ex group than in the control one $(P=0.036)$. Accordingly, the urinary albumin level was significantly lower in the SG-ex group than in the control one $(P=0 \cdot 044)$. These results suggest that SG-ex is likely to attenuate the kidney functions, which are often damaged under the case of the diabetic complication.

\section{Thiobarbituric acid-reactive substance level}

In order to analyse lipid peroxidation, TBARS levels of the liver, kidney, pancreas and plasma were measured (Table 2). TBARS level was standardised by malondialdehyde, a metabolite of oxidised lipid, and thus reflects the extent of lipid

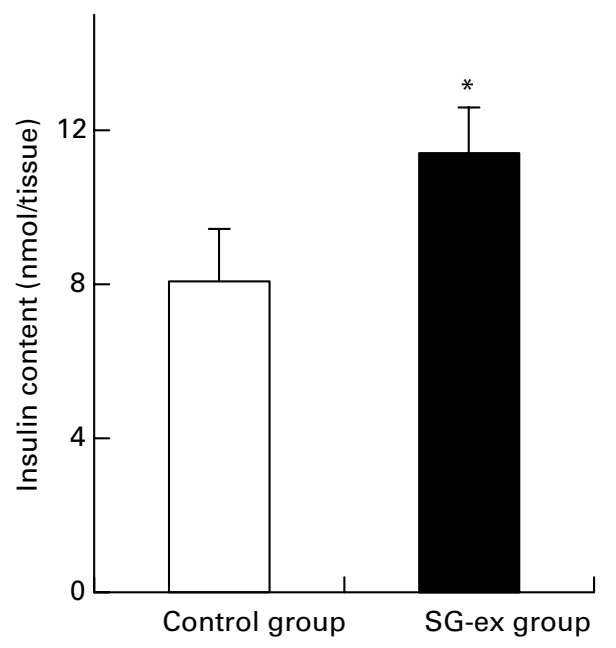

Fig. 3. Fasting pancreatic insulin levels measured by the sandwich ELISA. (ם), Siraitia grosvenori-extract (SG-ex)-supplemented group; ( $\square$ ), control group. Values are means ( $n$ 10), with standard errors represented by vertical bars. ${ }^{*}$ Mean value was significantly different from that of the control group $(P=0.013)$.
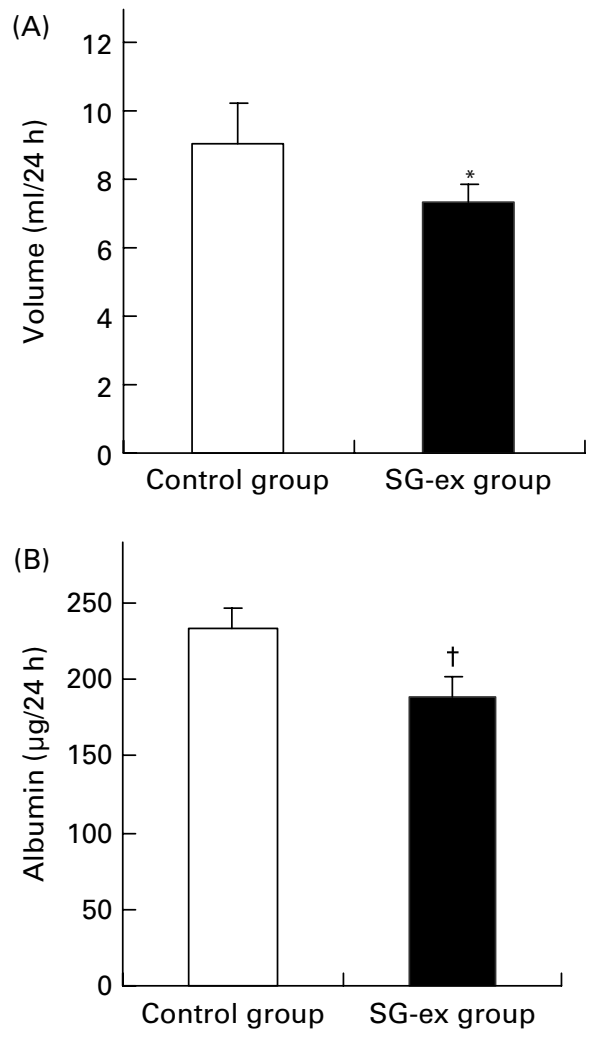

Fig. 4. Urine volume and urinary albumin levels. Urine was collected for $24 \mathrm{~h}$. Excreted urine volume (A) and urinary albumin levels (B) were measured for $2 \mathrm{~d}$ consecutively. ( $\square)$, Siraitia grosvenori-extract (SG-ex)-supplemented group; $(\square)$, control group. Values are means ( $n 10)$, with standard errors represented by vertical bars. *Mean value was significantly different from that of the control group $(P=0.036)$. †Mean value was significantly different from that of the control group $(P=0.044)$.

peroxidation. Average TBARS values were higher in control than in SG-ex-supplemented rats in all four tissues that we measured in the present study. In particular, TBARS values in both liver and plasma were significantly lower in SG-exsupplemented rats, suggesting that lipid peroxidation was inhibited by long-term administration of SG-ex.

\section{Plasma analysis}

Glutamic oxaloacetic transaminase and $\gamma$-glutamyl transpeptidase were significantly lower in the SG-ex group. In addition,

Table 2. Thiobarbituric acid-reactive substance (TBARS) levels (nmol/mg) in various tissues*

(Mean values with their standard errors)

\begin{tabular}{lcccccc}
\hline & \multicolumn{2}{c}{ Control $(n$ 10 $)$} & & \multicolumn{2}{c}{ SG-ex $(n$ 10) } & \\
\cline { 2 - 3 } & Mean & SEM & & Mean & SEM & $P$ \\
\hline Liver & 1.55 & 0.23 & & 0.77 & 0.02 & 0.007 \\
Plasma & 0.146 & 0.009 & & 0.113 & 0.014 & 0.017 \\
Pancreas & 58.5 & 2.9 & & 50.8 & 3.6 & 0.067 \\
Kidney & 0.72 & 0.08 & & 0.66 & 0.05 & 0.309 \\
\hline
\end{tabular}

SG-ex, Siraitia grosvenori Swingle extract.

${ }^{*}$ Rats were fed the control (without SG-ex) or the experimental (with SG-ex) diet for 13 weeks (see Table 1). TBARS levels of the liver, kidney, pancreas and plasma were determined. Results are expressed as a malondialdehyde equivalent (nmol malondialdehyde/mg protein). 
Table 3. Biochemical analysis of the plasma*

(Mean values with their standard errors)

\begin{tabular}{|c|c|c|c|c|c|}
\hline & \multicolumn{2}{|c|}{ Control (n 10) } & \multicolumn{2}{|c|}{ SG-ex ( $n$ 10) } & \multirow[b]{2}{*}{$P$} \\
\hline & Mean & SEM & Mean & SEM & \\
\hline Total cholesterol (mg/l) & 808 & 24 & 779 & 17 & 0.426 \\
\hline TAG $(\mathrm{mg} / \mathrm{l})$ & 411 & 37 & 391 & 26 & 0.332 \\
\hline GOT (IU/I) & $27 \cdot 5$ & 0.8 & $24 \cdot 8$ & 1.1 & 0.042 \\
\hline GPT (IU/I) & 6.9 & 0.3 & $6 \cdot 4$ & 0.2 & 0.093 \\
\hline$\gamma$-GTP (IU/I) & 3.3 & 0.1 & $2 \cdot 8$ & 0.1 & 0.011 \\
\hline LDH (IU/I) & $266 \cdot 2$ & $22 \cdot 5$ & $235 \cdot 0$ & 8.4 & 0.051 \\
\hline ALP (IU/I) & $148 \cdot 8$ & 8.2 & 137.6 & 4.0 & 0.368 \\
\hline
\end{tabular}

SG-ex, Siraitia grosvenori Swingle extract; GOT, glutamic oxaloacetic transaminase; GPT, glutamic pyruvic transaminase; $\gamma$-GTP, $\gamma$-glutamyl transpeptidase; $\mathrm{LDH}$, lactic dehydrogenase; ALP, alkaline phosphatase.

${ }^{*}$ Rats were fed the control (without SG-ex) or the experimental (with SG-ex) diet for 13 weeks (see Table 1)

average values of TAG, glutamic pyruvic transaminase, lactic dehydrogenase and alkaline phosphatase were lower in the SG-ex group (Table 3), although these differences were not statistically significant. These results suggest that the decline of liver function caused by diabetes is attenuated by longterm supplementation of SG-ex in rats.

\section{Discussion}

We demonstrated that a diet which is supplemented with SG-ex exerted antidiabetic effects that appear to moderately ameliorate various tissues from the typical diabetic pathological status. In addition, a 13-week supplementation of SG-ex did not show any adverse effects in GK rats, including feeding behaviour, body weight and various biochemical parameters in various organs.

We observed a trend of a lower $\mathrm{PG}_{\mathrm{NF}}$ in the SG-ex-supplemented rats compared with the control rats, but it did not reach the statistically significant level. The effect of long-term administration with voglibose, an $\alpha$-glucosidase inhibitor, on the GK rat has been reported (Wada et al. 1999). Treatment was started at 12 weeks of age and the $\mathrm{PG}_{\mathrm{NF}}$ become significantly lower in the voglibose-treated GK rat than that in the control GK rat at 24 weeks of age. Although the $\alpha$-glucosidase-inhibitory effect of SG-ex has been previously shown to be effective to inhibit the elevation of postprandial plasma glucose levels in Wistar rats (Suzuki et al. 2005), the activity of voglibose as an $\alpha$-glucosidase inhibitor is much more potent than that of SG-ex. Thus, it may take longer for SG-ex to significantly decrease the $\mathrm{PG}_{\mathrm{NF}}$.

In the present experiment, pancreatic insulin of adult GK rats at week 13 of the treatment (age 20 weeks) was $8 \cdot 1 \mathrm{nmol} / \mathrm{pan}$ creas, while the typical pancreatic insulin of adult Wistar rats (age 18 weeks) is $42.4 \mathrm{nmol} /$ pancreas (Movassat et al. 1997). We observed a significant increase of pancreatic insulin in the SG-ex-supplemented GK rat, although it was about $11.4 \mathrm{nmol} /$ pancreas and still much lower than the normal level in Wistar rats. The GK rat has been shown to develop an alteration of $\beta$-cells as early as embryonic day $21 \cdot 5$, and the deficit of total pancreatic $\beta$-cell mass in the GK rat has been shown to be maintained in the adult animal (Movassat et al. 1997). It is therefore possible that the $\beta$-cell functions are hardly recovered to the normal level after pancreatic $\beta$-cells were severely impaired during embryonic development.

Kidney function in streptozotocin-induced type 1 diabetic rats was shown to be disrupted and urinary albumin was reported to be increased in the diabetic rat (Adachi et al. 2000). Urine volume in the Wistar rat is reported to be about $6.9 \mathrm{ml} / 24 \mathrm{~h}$ (Adachi et al. 2000) and that in GK rats in the present study was $10.0 \mathrm{ml} / 24 \mathrm{~h}$. Thus, the urine volume of control GK rats being higher than that of normal Wistar rats causes the control GK rat to excrete more albumin. Therefore our observations, where SG-ex-supplemented GK rats excreted less urine and urinary albumin than control GK rats, suggest that kidney dysfunction caused by diabetes was attenuated by the supplementation with SG-ex.

The OGTT revealed that glucose tolerance in SG-ex-supplemented GK rats appeared to be ameliorated compared with that in control GK rats. The plasma glucose levels were decreased significantly $(P<0.05)$ at $120 \mathrm{~min}$ in SG-ex-supplemented GK rats. The plasma insulin levels in the SG-ex-supplemented GK rats at 15 min were significantly higher than that in the control rats. An antioxidant, $\alpha$-tocopherol, has been reported to ameliorate glycaemic control in GK rats (Ihara et al. 2000). It was reported that in the OGTT, plasma glucose levels were decreased significantly at 30 and $120 \mathrm{~min}$ in the $\alpha$-tocopherol-supplemented GK rats and plasma insulin levels in $\alpha$-tocopherol-supplemented GK rats were significantly higher at $30 \mathrm{~min}$, which is quite similar to what we have observed in the SG-ex-supplemented GK rats. 11OM-V, one of the sweet components in SG-ex, has been reported to have an antioxidative effect (Takeo et al. 2002), and thus it may be possible that antioxidative components in SG-ex such as 11OM-V could have a similar effect as $\alpha$-tocopherol.

It has been shown that the chronic hyperglycaemic state in the GK rat induces oxidative stress on the pancreatic $\beta$-cells, which appeared to cause cytotoxicity making pathological conditions worse (Ihara et al. 1999). $\alpha$-Tocopherol has been found to be accumulated in the pancreas when supplemented in the diet (Ihara et al. 2000). Although it has not yet been directly proven that antioxidative components in SG-ex are absorbed into the circulation, the reductions of lipid peroxidation (measured by TBARS) in the liver, plasma and pancreas of SG-ex-supplemented GK rats suggest that antioxidative components are absorbed and delivered to various tissues. It is therefore possible that antioxidative components in SG-ex were targeted to the pancreas and helped to repair its function as well as $\alpha$-tocopherol did. This feasible hypothesis still needs to be investigated.

In a recent placebo-controlled large-scale clinical trial, acarbose, an $\alpha$-glucosidase inhibitor, has been shown to improve sensitivity to insulin and decrease postprandial hyperglycaemia, thereby releasing the stress on the $\beta$-cells (Chiasson et al. 1996). The fundamental mechanisms of acarbose and that of SG-ex are similar, i.e. they act as $\alpha$-glucosidase inhibitors. Therefore it is conceivable that long-term supplementation with SG-ex could have the similar antidiabetic effect in human subjects.

In summary, SG-ex exhibited an antidiabetic effect on the spontaneously diabetic GK rat by improving insulin response in the OGTT, accumulating insulin in the pancreas in the fasting state, ameliorating kidney function, and enhancing antioxidative properties in the liver and the plasma. 


\section{References}

Adachi T, Yasuda K, Okamoto Y, et al. (2000) T-1095, a renal $\mathrm{Na}^{+}$glucose transporter inhibitor, improves hyperglycemia in streptozotocin-induced diabetic rats. Metabolism 49, 990-995.

Bisbis S, Bailbe D, Tormo MA, Picarel-Blanchot F, Derouet M, Simon J \& Portha B (1993) Insulin resistance in the GK rat: decreased receptor number but normal kinase activity in the liver. Am J Physiol 265, E807-E813.

Chiasson JL, Josse RG, Leiter LA, et al. (1996) The effect of acarbose on insulin sensitivity in subjects with impaired glucose tolerance. Diabetes Care 19, 1190-1193.

Clissold SP \& Edwards C (1988) Acarbose, a preliminary review of its pharmacodynamic and pharmacokinetic properties, and therapeutic potential. Drugs 35, 214-243.

Goto Y \& Kakizaki M (1981) The spontaneous-diabetes rat: a model of noninsulin-dependent diabetes mellitus. Proc Jpn Acad 57, 381-384.

Goto Y, Kakizaki M \& Masaki N (1975) Spontaneous diabetes produced by selective breeding of normal Wistar rats. Proc Jpn Acad 51, 80-85.

Hossen MA, Shinmei Y, Jiang S, Takubo M, Tsumuro T, Murata Y, Suguira M \& Kamei C (2005) Effect of Lo Han Kuo (Siraitia grosvenori Swingle) on nasal rubbing and scratching behavior in ICR mice. Biol Pharm Bull 28, 238-241.

Ihara Y, Toyokuni S, Uchida K, Odaka H, Tanaka T, Ikeda H, Hiai H, Seino Y \& Yamada Y (1999) Hyperglycemia causes oxidative stress in pancreatic $\beta$-cells of GK rats, a model of type 2 diabetes. Diabetes 48, 927-932.

Ihara Y, Yamada Y, Toyokuni S, et al. (2000) Antioxidant $\alpha$-tocopherol ameliorates glycemic control of GK rats, a model of type 2 diabetes. FEBS Lett 473, 24-46.

Janssen U, Phillips AO \& Floege J (1999) Rodent models of nephropathy associated with type II diabetes. J Nephrol 12, 159-172.

Kasai R, Nie RL, Nashi K, Ohtani K, Zhou J, Tao GD \& Tanaka O (1989) Sweet cucurbitane glycosides from fruits of Siraitia samensis (chi-zi luo-han-guo), a Chinese folk medicine. Agric Biol Chem 53, 3347-3349.

Lee C (1975) Intense sweetener from lo han kuo (Momordica grosvenori). Experientia 31, 533-534.

Matsumoto K, Kasai R, Ohtani K \& Tanaka O (1990) Minor cucurbitane-glycosides from fruits of Siraitia grosvenori (Cucurbitaceae). Chem Pharm Bull 38, 2030-2032.

Movassat J, Saulnier C, Serradas P \& Portha B (1997) Impaired development of pancreatic $\beta$-cell mass is a primary event during the progression to diabetes in the GK rat. Diabetologia 40, 916-925.

National Research Council (1985) Guide for the Care and Use of Laboratory Animals. Publication no. 85-23, revised ed.Bethesda, MA: National Institute of Health.
Ohkawa H, Ohishi N \& Yagi K (1979) Assay for lipid peroxides in animal tissue by thiobarbituric acid reaction. Anal Biochem 95, $351-358$.

O'Rourke CM, Davis JA, Saltiel AR \& Cornicell JA (1997) Metabolic effects of troglitazone in the Goto-Kakizaki rat, a nonobese and normolipidemic rodent model of non-insulin-dependent diabetes mellitus. Metabolism 46, 192-198.

Ostenson CG, Khan A, Abdel-Halim SM, Guenifi A, Suzuki K, Goto Y \& Efendic S (1993) Abnormal insulin secretion and glucose metabolism in pancreatic islets from the spontaneously diabetic GK rat. Diabetologia 36, 3-8.

Portha B, Serradas P, Bailbe D, Suzuki K, Goto Y \& Giroix MH (1991) $\beta$ Cell insensitivity to glucose in GK rat, a spontaneous non-obese model for type 2 (non insulin-dependent) diabetes. Diabetes 40, 486-496.

Rury RH, Robert CT \& Carole AC (1999) A randomized doubleblind trial of acarbose in type 2 diabetes shows improved glycemic control over 3 years (U.K. Prospective Diabetes Study 44). Diabetes Care 22, 960-964.

Saito N, Sakai H, Sekihara H \& Yajima Y (1998) Effect of an $\alpha$-glucosidase inhibitor (voglibose), in combination with sulponilureas, on glycemic control in type 2 diabetes patients. J Intern Med Res 26, 219-232.

Suzuki YA, Murata Y, Inui H, Sugiura M \& Nakano Y (2005) Triterpene glycosides of Siraitia grosvenori inhibit rat intestinal maltase and suppress the rise in plasma glucose level after single oral administration of maltose in rats. J Agric Food Chem 53, 2941-2946.

Takasaki M, Konoshima T, Murata Y, Sugiura M, Nishino H, Tokuda H, Matsumoto K, Kasai R \& Yamasaki K (2003) Anticarcinogenic activity of natural sweeteners, cucurbitane glycosides, from Momordica grosvenori. Cancer Lett 198, 37-42.

Takemoto T, Arihara S, Nakajima T \& Okuhira M (1983) Studies on the constituents of Fructus momordicae. I. On the sweet principle. Yakugaku Zasshi 11, 1151-1154.

Takeo E, Yoshida H, Tada N, et al. (2002) Sweet element of Siraitia grosvenori inhibit oxidative modification of low-density lipoprotein. J Atherosclerosis Thromb 9, 114-120.

Toeller M (1994) $\alpha$-Glucosidase inhibitors in diabetes: efficacy in NIDDM subjects. Eur J Clin Invest 24, Suppl. 3, 31-35.

Wada R, Koyama M, Mizukami H, Odaka H, Ikeda H \& Yagihashi S (1999) Effects of long-term treatment with $\alpha$-glucosidase inhibitor on the peripheral nerve function and structure in Goto-Kakizaki rats: a genetic model for type 2 diabetes. Diabetes Metab Res Rev 15, 332-337.

Zhou YP, Ostenson CG, Ling ZC \& Grill V (1995) Deficiency of pyruvate dehydrogenase activity in pancreatic islets of diabetic GK rats. Endocrinology 136, 3546-3551. 